\title{
CORRECTION
}

View Article Online

View Journal I View Issue

Check for updates

Cite this: Soft Matter, 2021, 17, 4213

DOI: $10.1039 / \mathrm{d} 1 \mathrm{sm} 90049 \mathrm{~h}$

rsc.li/soft-matter-journal

\section{Correction: A neural network-based algorithm for high-throughput characterisation of viscoelastic properties of flowing microcapsules}

\author{
Tao Lin, ${ }^{a}$ Zhen Wang, ${ }^{b}$ Wen Wang ${ }^{a}$ and Yi Sui ${ }^{a}$
}

Correction for 'A neural network-based algorithm for high-throughput characterisation of viscoelastic properties of flowing microcapsules' by Tao Lin et al., Soft Matter, 2021, DOI: 10.1039/d0sm02121k.

The authors wish to include a citation to 'A high-throughput method to characterize membrane viscosity of flowing microcapsules' by Tao Lin et al., Physics of Fluids, 2021, DOI: 10.1063/5.0031640. ${ }^{1}$ They also wish to provide the following clarification for readers:

The work reported in the original article in Soft Matter was carried out in parallel to our recent work in Physics of Fluids (POF). Two different types of neural-network algorithms are reported in the two works that make predictions with different mechanisms. The POF article is based on a convolutional neural network (CNN), while the Soft Matter article combines parts of a CNN with a long short-term memory network (i.e., a hybrid model). A significant advantage/advance of the hybrid model is that it deals with individual instantaneous images of the capsule and builds their temporal connections. It can therefore be much more accurate. However, the CNN (in the POF work) has a merit of being simple.

When analysing the performance of the two methods, similar procedures were followed, and some data of capsule profiles for training and testing the networks were shared. Therefore, some similarities can be seen in the figures of the two papers. In Fig. 3 in the Soft Matter paper and Fig. 5 of the POF paper, and also Fig. 4 of the Soft Matter paper and Fig. 6 of the POF paper, the results regarding performance of the two networks are different by 2-6\%. However, these procedures and capsule data are not the main points/results of the papers.

The Royal Society of Chemistry apologises for these errors and any consequent inconvenience to authors and readers.

\section{References}

1 T. Lin, Z. Wang, R. Lu, W. Wang and Y. Sui, Phys. Fluids, 2021, 33, 011906, DOI: 10.1063/5.0031640.

\footnotetext{
${ }^{a}$ School of Engineering and Materials Science, Queen Mary University of London, London E1 4NS, UK. E-mail: y.sui@qmul.ac.uk

${ }^{b}$ Department of Mechanical Engineering, University College London, London WC1E 6BT, UK
} 